\title{
In-flight PSF calibration of the NuSTAR hard X-ray optics
}

An, Hongjun; Madsen, Kristin K.; Westergaard, Niels J.; Boggs, Steven E.; Christensen, Finn E.; Craig, William W.; Hailey, Charles J.; Harrison, Fiona A.; Stern, Daniel K.; Zhang, William W.

\section{Published in:}

Proceedings of the SPIE Astronomical Telescopes + Instrumentation 2014: Ultraviolet to Gamma Ray

Link to article, DOI:

$10.1117 / 12.2055481$

Publication date:

2014

Document Version

Publisher's PDF, also known as Version of record

Link back to DTU Orbit

Citation (APA):

An, H., Madsen, K. K., Westergaard, N. J., Boggs, S. E., Christensen, F. E., Craig, W. W., Hailey, C. J., Harrison, F. A., Stern, D. K., \& Zhang, W. W. (2014). In-flight PSF calibration of the NuSTAR hard X-ray optics. In T. Takahashi, J-W. A. den Herder, \& M. Bautz (Eds.), Proceedings of the SPIE Astronomical Telescopes + Instrumentation 2014: Ultraviolet to Gamma Ray (Vol. 9144). [ 91441Q] SPIE - International Society for Optical Engineering. Proceedings of SPIE - The International Society for Optical Engineering https://doi.org/10.1117/12.2055481

\section{General rights}

Copyright and moral rights for the publications made accessible in the public portal are retained by the authors and/or other copyright owners and it is a condition of accessing publications that users recognise and abide by the legal requirements associated with these rights.

- Users may download and print one copy of any publication from the public portal for the purpose of private study or research.

- You may not further distribute the material or use it for any profit-making activity or commercial gain

- You may freely distribute the URL identifying the publication in the public portal 


\title{
In-flight PSF calibration of the NuSTAR hard X-ray optics
}

\author{
Hongjun $\mathrm{An}^{a}$, Kristin K. Madsen ${ }^{b}$, Niels J. Westergaard ${ }^{c}$, Steven E. Boggs ${ }^{d}$, Finn E. \\ Christensen $^{c}$, William W. Craig ${ }^{d, e}$, Charles J. Hailey ${ }^{f}$, Fiona A. Harrison ${ }^{b}$, Daniel K. Stern ${ }^{g}$, \\ William W. Zhang ${ }^{h}$ \\ ${ }^{a}$ Department of Physics, McGill University, Montreal, Quebec, H3A 2T8, Canada; \\ ${ }^{b}$ Cahill Center for Astronomy and Astrophysics, California Institute of Technology, Pasadena, \\ CA 91125, USA; \\ ${ }^{c}$ DTU Space, National Space Institute, Technical University of Denmark, Elektrovej 327, \\ DK-2800 Lyngby, Denmark; \\ ${ }^{d}$ Space Sciences Laboratory, University of California, Berkeley, CA 94720, USA; \\ ${ }^{e}$ Lawrence Livermore National Laboratory, Livermore, CA 94550, USA; \\ ${ }^{f}$ Columbia Astrophysics Laboratory, Columbia University, New York NY 10027, USA; \\ ${ }^{g}$ Jet Propulsion Laboratory, California Institute of Technology, Pasadena, CA 91109, USA; \\ ${ }^{h}$ Goddard Space Flight Center, Greenbelt, MD 20771, USA
}

\begin{abstract}
We present results of the point spread function (PSF) calibration of the hard X-ray optics of the Nuclear Spectroscopic Telescope Array (NuSTAR). Immediately post-launch, NuSTAR has observed bright point sources such as Cyg X-1, Vela X-1, and Her X-1 for the PSF calibration. We use the point source observations taken at several off-axis angles together with a ray-trace model to characterize the in-orbit angular response, and find that the ray-trace model alone does not fit the observed event distributions and applying empirical corrections to the ray-trace model improves the fit significantly. We describe the corrections applied to the ray-trace model and show that the uncertainties in the enclosed energy fraction (EEF) of the new PSF model is $\lesssim 3 \%$ for extraction apertures of $R \gtrsim 60^{\prime \prime}$ with no significant energy dependence. We also show that the PSF of the NuSTAR optics has been stable over a period of $\sim 300$ days during its in-orbit operation.
\end{abstract}

Keywords: NuSTAR, X-ray optics, Point Spread Function (PSF), Half Power Diameter (HPD), Calibration

\section{INTRODUCTION}

The Nuclear Spectroscopic Telescope Array (NuSTAR) is the first hard X-ray focusing telescope on orbit (Fig. 1 left), operating in the 3-79 keV band. ${ }^{1,2}$ NUSTAR has two hard X-ray focusing optics and focal plane modules (FPMA and FPMB). The hard X-ray optics employed in NuSTAR are segmented glass optics ${ }^{3}$ which are composed of axially and azimuthally segmented mirrors stacked on a Titanium mandrel. ${ }^{4}$ Cylindrical mirrors are formed by thermally slumping flat glass onto a precision mandrel. ${ }^{5}$ The mirrors are coated with multilayer ${ }^{6}$ and then bonded on precisely machined graphite spacers to form a conic approximation to the Wolter-I optics (Fig. 1 right). Each optic has 133 shells; the inner 90 shells are coated with Pt/C multilayer and the outer 43 shells with $\mathrm{W} / \mathrm{Si}^{7}$

The good angular response of the optics is crucially important not only to study extended sources such as supernova remnants (SNRs) and pulsar wind nebulae (PWNe) but also to have better sensitivity for point source detection. Furthermore, accurately measuring the enclosed energy fraction (EEF) with radius and energy is very important since it not only determines the absolute normalization but may change the slope of Ancillary Response File (ARF). PSF of the NuSTAR optics is mainly dominated by the glass figure error and alignment

Further author information: (Send correspondence to H. An)

H. An: E-mail: hjan@physics.mcgill.ca, Telephone: 15143986820

Space Telescopes and Instrumentation 2014: Ultraviolet to Gamma Ray, edited by Tadayuki Takahashi, Jan-Willem A. den Herder, Mark Bautz, Proc. of SPIE Vol. 9144, 91441Q · @ 2014 SPIE CCC code: $0277-786 \mathrm{X} / 14 / \$ 18 \cdot$ doi: $10.1117 / 12.2055481$

Proc. of SPIE Vol. $914491441 \mathrm{Q}-1$ 

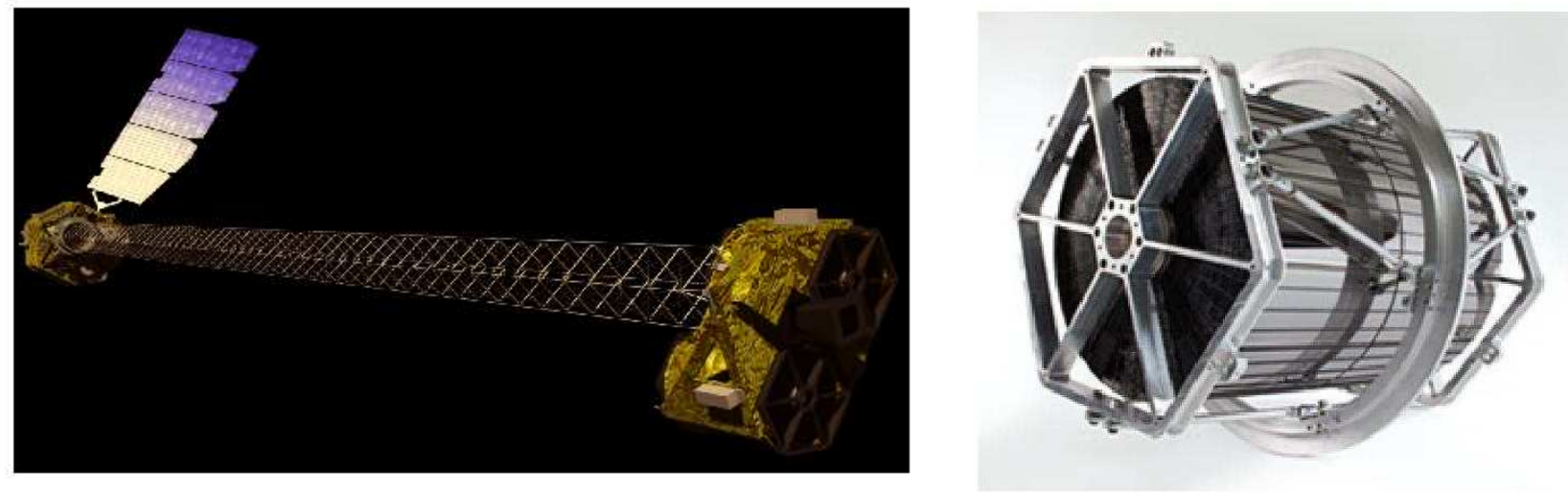

Figure 1. The NuSTAR observatory (left) and the hard X-ray optic (right). The optic is $\sim 50 \mathrm{~cm}$ long and its focal length is $\sim 10 \mathrm{~m}$.

of the mirror layers. Large scale figure errors $(\gtrsim \mathrm{cm})$ in the mirror are efficiently suppressed during the mounting process although it may introduce some large scale figure errors by forcing cylindrical mirrors to conical shapes. The small scale figure errors $(\lesssim \mathrm{cm})$ are hard to suppress and may be added as distortion during mounting. The alignment between mirror shells was maintained by precisely machining the spacers to the proper conical shape for each shell; any errors in the machining will cause image blurring. During the optics assembly, the alignment was monitored, and the glass figures were measured.

Data for estimating the NuSTAR PSF on ground were taken with a laser metrology system, a mechanical probe, and the X-ray calibration facility. ${ }^{8}$ The data were combined into a ray-trace model and used to derive the PSF of the optics, and the Half Power Diameter (HPD) was estimated to be $52^{\prime \prime}$ with an uncertainty of $\sim 4^{\prime \prime}{ }^{9}$ The ground calibration was sufficient to ensure that the optics were of the required quality, but because of the finite beam-line length and systematics in the measurements, it is necessary to validate the PSF's in-orbit performance using bright point sources. We focus on the PSF calibration of the $N u S T A R$ optics in this paper, and more comprehensive calibration of the observatory is presented elsewhere (Madsen et al. 2014, in prep.). We present the observations and data processes in Section 2, and data analyses and the results in Section 3, and then conclude in Section 4.

\section{OBSERVATIONS}

We use bright point source observations to model the in-flight PSF. The sources used for PSF calibration are listed in table 1 . The observations span a time interval of $\sim 300$ days, and hence we can see how and if the PSF of the optics changes over the interval. The off-axis angle in an observation can change by $\sim 1^{\prime}$ because of the lack of pointing stability (e.g., relative motion between the optics and the detector benches), ${ }^{1}$ and we report the average value of off-axis angle $(\theta)$ distribution of the observations in Table 1 . Since the width of the off-axis angle distribution is not large and the $N u S T A R$ PSF does not rapidly change with off-axis angle, we grouped the observations into two: one having the average off-axis angle $\lesssim 2$ ' for the 'near' on-axis PSF calibration and the other for the off-axis PSF.

We used observations 1 and 2 in Table 1 for the off-axis PSF calibration (Section 3.2), and all the rest for the 'near' on-axis PSF (Section 3.1), and compared the event distribution of observation 5 with those of observations 7, 8, 10 and 11 in order to check the stability of the PSF over time (Section 3.4). We processed the observations with nupipeline of NUSTARDAS v1.2.0 using standard filtering procedure along with CALDB 20130509 to produce cleaned event files. We further processed the cleaned event files for the analyses as described below. 
Table 1. Observations used for PSF calibration

\begin{tabular}{lcccccc}
\hline Obs. \# & Source & Obs. ID & $\begin{array}{c}\text { Obs. Date } \\
(\mathrm{UTC})\end{array}$ & $\begin{array}{c}\text { Exposure } \\
(\mathrm{ks})\end{array}$ & $\begin{array}{c}\text { Off-axis angle }^{\mathrm{a}} \\
\text { (arcminutes) }^{2}\end{array}$ & Comment \\
\hline \hline 1 & Cyg X-1 & 00001007001 & $2012-06-28$ & 2.4 & 3 & For off-axis PSF \\
2 & Cyg X-1 & 00001008001 & $2012-06-28$ & 4.3 & 3 & For off-axis PSF \\
3 & GRS 1915+105 & 10002004001 & $2012-07-03$ & 15 & 1 & \\
4 & Cyg X-1 & 10002003001 & $2012-07-06$ & 9 & 0.5 & \\
5 & Vela X-1 & 10002007001 & $2012-07-09$ & 11 & 1 & \\
6 & GS0834 & 10002018001 & $2012-07-11$ & 31 & 1 & \\
7 & Her X-1 & 30002006002 & $2012-09-19$ & 28 & 2 & \\
8 & Her X-1 & 30002006005 & $2012-09-22$ & 22 & 1 & \\
9 & Her X-1 & 30002006007 & $2012-09-24$ & 27 & 1 & \\
10 & Vela X-1 & 30002007002 & $2013-04-22$ & 7 & 1 & \\
11 & Vela X-1 & 30002007003 & $2013-04-22$ & 24 & 2 & \\
\hline
\end{tabular}

${ }^{\text {a }}$ Average value for a distribution.

\section{DATA ANALYSIS AND RESULTS}

\subsection{Near On-axis PSF}

A ray-trace model was developed prior to launch. ${ }^{10}$ The model uses the surface figures of the mirrors measured with the mechanical probe scans after mounting, ${ }^{7}$ and the reflectivity measurements made with a sample of multilayer-coated mirrors ${ }^{6}$ as inputs, to trace X-ray photons down to the focal plane detectors, producing the 2$\mathrm{D}$ event distribution, ray-traced PSF. We generated ray-traced PSFs at every $0.5^{\prime}$ out to $8.5^{\prime}$, shown in Figure 2 left. In the Figure, we shifted the PSF for an off-axis angle to the positive x-axis by the off-axis angle, and azimuthally rotated the $\operatorname{PSF}\left(\phi_{\text {off }}=0-300^{\circ}\right.$ with a step of $\left.60^{\circ}\right)$ to show the 2 -D map. The Figure shows that the PSF is very circular out to $\theta \sim 2^{\prime}$, and gradually distorts to a bowtie shape at large off-axis angles.

For comparison with the ray-traced PSF, we extracted events from the point source observations (see Table 1) in several energy ranges (3-4.5 keV, 4.5-6 keV, 6-8 keV, 8-12 keV, 12-20 keV and 20-79 keV), and produced observed radial profiles centered at the intensity peak for each observation. Note that the ray-traced PSF does not have the energy dependence because we do not have the ground measurements below $\sim 8 \mathrm{keV} .{ }^{9}$ However, we resolved the event distribution in the energy space in order to see any change of the distribution with energy. Since the off-axis angle was changing during an observation, we weighted the ray-traced PSF with the aspect solution and vignetting function of the observation. The model PSF is formulated as

$$
\Psi(x, y)=\int \Psi_{\mathrm{RT}}\left(x-x_{s}^{*}, y-y_{s}^{*}, x_{s}(t), y_{s}(t)\right) \times \mathcal{V}\left(x_{s}(t), y_{s}(t)\right) d t \times \mathcal{E}\left(x-x_{s}^{*}, y-y_{s}^{*}\right),
$$

where $\Psi(x, y)$ is the PSF model, $\Psi_{\mathrm{RT}}$ is the ray-traced PSF, $x_{s}^{*}$ and $y_{s}^{*}$ are the sky positions of the source which are constant in time, $x_{s}(t)$ and $y_{s}(t)$ are the source positions with respect to the optical axis which determine the off-axis angle $\theta$ and $\phi_{\text {off }}, \mathcal{V}\left(x_{s}(t), y_{s}(t)\right)$ is the vignetting factor for the off-axis angle, and $\mathcal{E}\left(x-x_{s}^{*}, y-y_{s}^{*}\right)$ is the exposure map for the observation. For source positions $x_{s}(t)$ and $y_{s}(t)$ at a time $t$, the ray-traced PSF corresponding to $\theta$ is read in from the database and is rotated by $\phi_{\text {off }}$ because the ray-trace PSFs are stored for different $\theta$ values, but not for different $\phi_{\text {off }}$ in the database. Note that the point source exposure is almost flat, and the overall shape of the PSF does not change much by multiplying the exposure. However, it is important for some localized effects such as detector gap.

Since the sources were very bright and we do not have a large enough region for background extraction in the detector, the background was generated using a background model. ${ }^{11}$ The background model was constructed considering the internal, the aperture and the cosmic X-ray background (CXB) components. The internal background is spatially uniform at low energies but less uniform at high energies. The aperture component is nonuniform and mostly in the low energy band. These two components do not go through the optics, and thus 

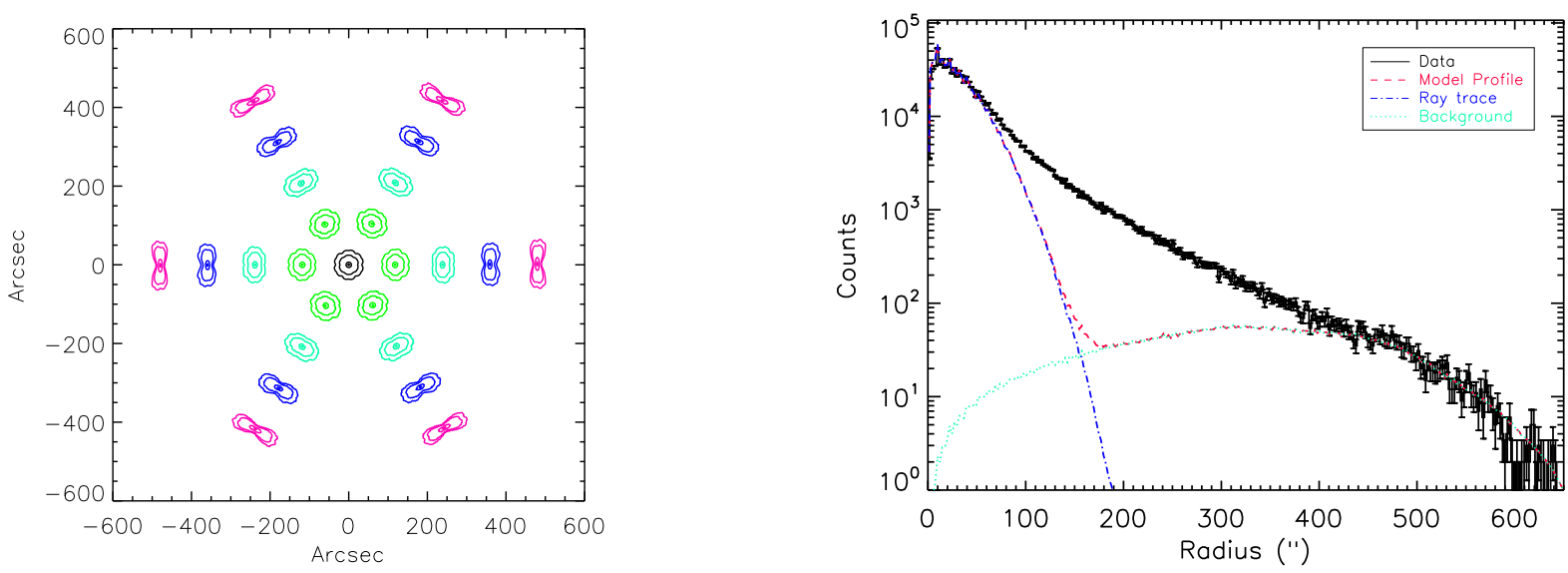

Figure 2. Left: 2-D ray-trace PSF shapes at off-axis angles $\theta$ of $0^{\prime}-8^{\prime}$ with a step of $2^{\prime}$. Each color represents PSF for an off-axis angle. Off-axis PSFs were shifted to the positive $\mathrm{x}$-axis by the off-axis angle, and azimuthally rotated by $60^{\circ}-300^{\circ}$. Right: Radial profile of the Cyg X-1 observation in the $5-8 \mathrm{keV}$ band. Best-fit model (red) was constructed with the ray-trace PSF (blue, Equation 1) and background (cyan).

are unfocused. The CXB component goes through the optics and is focused. The backgrounds were very small compared to the source, dominating only above $\mathrm{R}>400^{\prime \prime}$ (see Fig. 3 ).

We fit the observed radial profiles with those of the model PSF in Equation 1 and the background, adjusting the normalization constants only. An example of the fit is shown in Figure 2 right. After comparing with the near on-axis observations, we found that the PSF model in Equation 1 does not describe the observed event distributions. Specifically, we find that (i) there is a broad wing at $R=100-400^{\prime \prime}$ in the radial profiles of the observations, (ii) the central cores of observed radial profiles are slightly broader than that of the model, which cannot be explained with the current PSF model (see Fig. 2). We therefore modified the PSF model.

In order to remove the large residuals in the wing $\left(R=100-400^{\prime \prime}\right)$, we added an exponential function to the PSF in Equation 1. The 2-D wing component is expressed in the following formula:

$$
\Psi_{W}(x, y)=\int e^{-a \sqrt{\left(x-x_{s}^{*}\right)^{2}+\left(y-y_{s}^{*}\right)^{2}}} \times \mathcal{V}\left(x_{s}(t), y_{s}(t)\right) d t \times \mathcal{E}\left(x-x_{s}^{*}, y-y_{s}^{*}\right) .
$$

Note that the shape of the exponential function does not change with the off-axis angle at this point. However, we further modify it below based on the off-axis observations (Section 3.2). For the core broadening, we used a Gaussian convolution model:

$$
\Psi_{G}(x, y)=\int \Psi_{\mathrm{RT}}\left(x-x_{s}^{*}, y-y_{s}^{*}, x_{s}(t), y_{s}(t)\right) \otimes G\left(x-x_{s}^{*}, y-y_{s}^{*}, \sigma\right) \times \mathcal{V}\left(x_{s}(t), y_{s}(t)\right) d t \times \mathcal{E}\left(x-x_{s}^{*}, y-y_{s}^{*}\right),
$$

where $G\left(x-x_{s}^{*}, y-y_{s}^{*}, \sigma\right)$ is a Gaussian function with width $\sigma$. The modified PSF is a combination of Equations 2 and 3 .

We then added the background component, produced the model radial profile, and fit the observed radial profile of each observation. The fitting parameters are the amplitude for the ray-traced PSF $\left(C_{0}\right)$, width of the Gaussian convolution core $(\sigma)$, amplitude and decay constant for the exponential wing $\left(C_{1}\right.$ and $\left.a\right)$, and amplitude for the background $\left(C_{2}\right)$. Note that we also included the off-axis correction (see Section 3.2) in the PSF model. Since fitting the observations simultaneously was not possible because they have different aspect history and background, we calculated $\chi^{2}$ for various values of fitting parameters for each observation, and found a set of parameters that minimized the combined $\chi^{2}$.

An example the observed radial profile for the Cyg X-1 observation (obs. 4) and the best-fit PSF model are shown in Figure 3. The wing correction in Equation 2 compensates for the large difference between the data and 

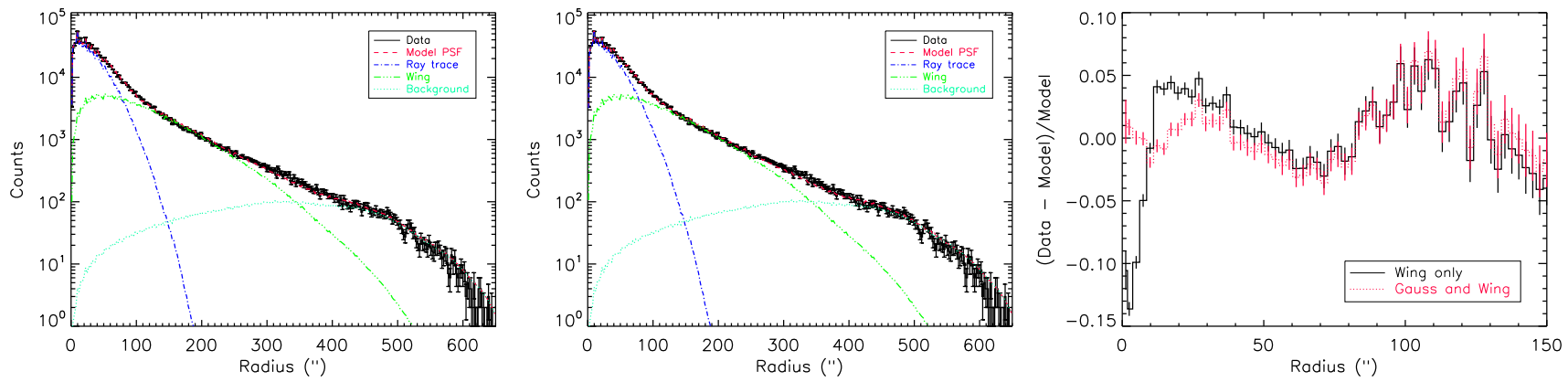

Figure 3. Left: Radial profile (black) of the Cyg X-1 observation in the 3-4.5 keV band and the best-fit function. The best-fit function (red) is a combination of the ray-trace PSF (blue, Equation 1), the wing component (green, Equation 2), and the background (cyan). Middle: Same as the left panel but with a different fit function which is a combination of the ray-trace PSF with the Gaussian convolution (Equation 3), the wing component (Equation 2), and the background. Right: Residuals after the fits for the left panel (black) and the middle panel (red).

the model seen in Figure 2, but there are still large residuals near the core $R \lesssim 15^{\prime \prime}$ (see Figs. 3 left and right). The large residuals near the core are removed when applying the core correction (Eq. 3, Fig. 3 middle and right). We note that there are large scale residuals with amplitude of $\sim 5 \%$ even after applying all the corrections above (see the red curve in Fig. 3 right). It was difficult to remove the large scale residuals for all the nine observations with a common function because the residuals differ from observation to observation. Therefore, we included the residuals in the error estimation for the EEF in Section 3.3.

\subsection{Off-axis PSF}

The radial profile of the NUSTAR PSF does not change much with the off-axis angle, i.e., the FWHM remains roughly constant, but the 2-D shape of the PSF gradually distorts with increasing off-axis angle due to geometrical shadowing of the shells, for example. As a result, the off-axis PSFs appear to be elongated as shown in Figure 2 left, and a 2-D off-axis PSF model correction is required. Since accurately modeling the PSF at large off-axis angles is difficult due to the paucity of counts, we matched the 2-D contours of the observations and the PSF in order to obtain off-axis correction factors.

The 2-D distortion due to the shadowing is already considered in the ray-traced model. However, the exponential wing is not yet incorporated into the ray-trace model, and thus we need to use a 2-D analytic distortion model for the wing component. Reduction in the effective area for a shell of a Wolter-I optic has been studied $^{12,13}$ and can be approximated with the following expression: ${ }^{12}$

$$
A_{\mathrm{eff}}(\theta) \approx A\left(1-\frac{2 \theta}{3 \alpha}\right) R(\alpha, \lambda)
$$

where $\theta$ is the off-axis angle, $\alpha$ is the grazing incidence angle of on-axis photons, $R(\alpha, \lambda)$ is the reflectivity and $\lambda$ is the photon wavelength. Note that azimuthal integration was performed in the above formula while we need the azimuth angle dependence in order to have a 2-D shape. Furthermore, we need to have an analytic expression for the reflectivity, and integrate over the 133 layers of the NuSTAR optics, which makes a detailed analytic implementation impossible.

Instead, we assumed that $A_{\mathrm{eff}}$ is a linear function of $\theta$, similar to the above, and added the $\phi=\tan ^{-1}((\mathrm{y}-$ $\left.\left.\mathrm{y}_{\mathrm{s}}^{*}\right) /\left(\mathrm{x}-\mathrm{x}_{\mathrm{s}}^{*}\right)\right)$ dependence as $\theta=\theta \cos ^{2}(\phi)$, where we use $\cos ^{2}(\phi)$ instead of $\cos (\phi)$ for the $\phi$ dependence in order to avoid $A_{\text {eff }}$ being greater than 1 , the on-axis value, in the left half plane (negative $x-x_{s}^{*}$ ). Note that $\phi$ is calculated for each sky pixel $x$ and $y$, and is different from the azimuthal angle of the source $\phi_{\text {off }}=\operatorname{atan}^{-1}\left(\mathrm{y}_{\mathrm{s}}(\mathrm{t}) / \mathrm{x}_{\mathrm{s}}(\mathrm{t})\right)$. In addition, we included an ellipticity factor in order to model the elongation of PSF at off-axis angles along the direction transverse to the off-axis angle (see Fig. 2 left), and used the following formulae to adjust the exponential wing at off-axis angles:

$$
A_{\mathrm{eff}}(x, y, \theta(t))=1-A(\theta(t)) \cos ^{2}\left(\tan ^{-1}\left(B(\theta(t))\left(y-y_{s}^{*}\right) /\left(x-x_{s}^{*}\right)\right)\right),
$$



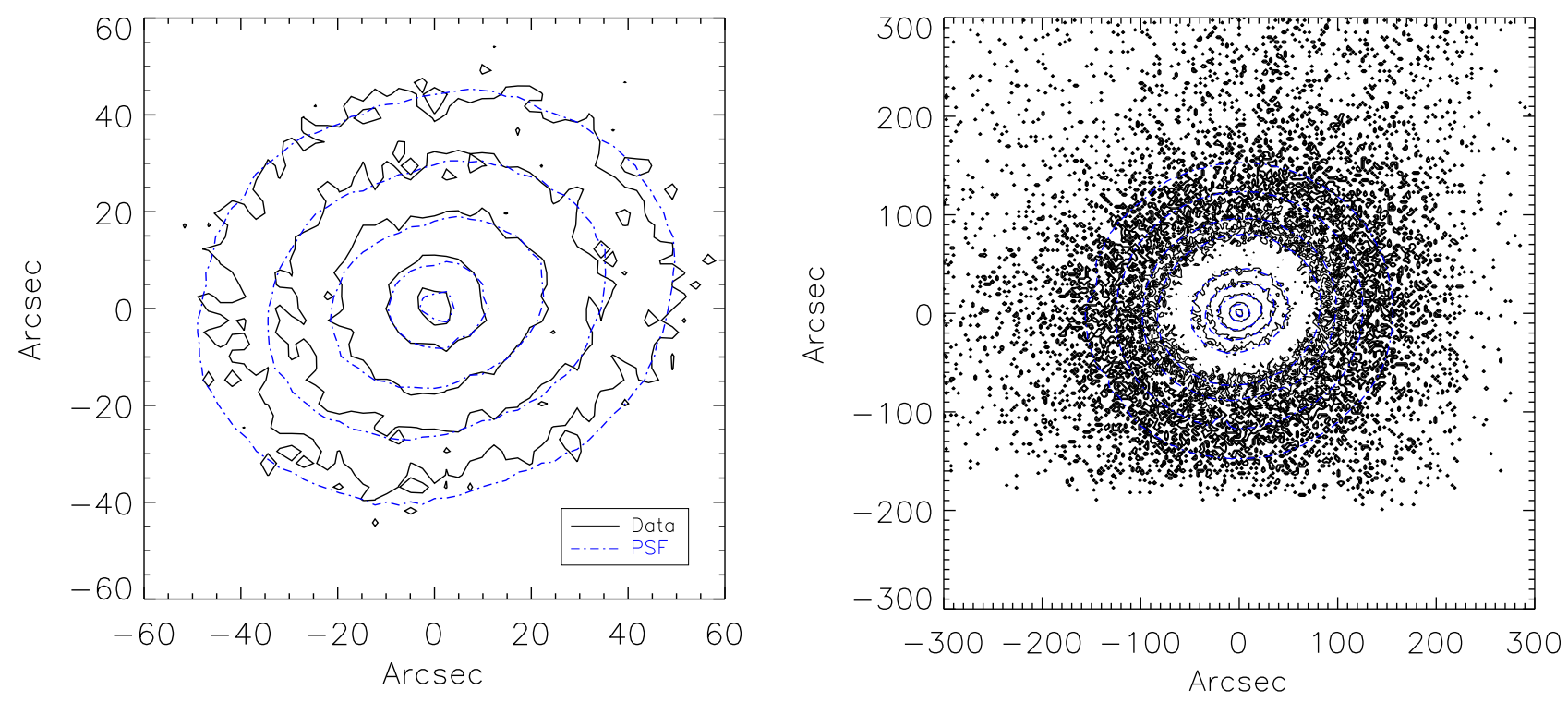

Figure 4. Count contours of observation 1 (black) and the model PSF (blue) for an off-axis angle $\theta \sim 3^{\prime}$ in a $2^{\prime} \times 2^{\prime}$ field (left) and in a $10^{\prime} \times 10^{\prime}$ field (right).

and

$$
F_{\text {wing }}\left(x, y, \theta(t), \phi_{\text {off }}(t)\right)=\mathcal{R}\left(\phi_{\text {off }}(t)\right)\left(e^{-a \sqrt{\left(x-x_{s}^{*}\right)^{2}+B(\theta)\left(y-y_{s}^{*}\right)^{2}}} \times A_{\text {eff }}(x, y, \theta(t))\right),
$$

where $A(\theta)$ is a linear function which is 0 when $\theta=0, B(\theta)$ is the ellipticity factor which is 1 when $\theta=0$, having the largest $A_{\text {eff }}$ and no elongation for the on-axis PSF, and $R\left(\phi_{\text {off }}(t)\right)$ is a rotation of a function around the axis perpendicular to the $x-y$ plane centered at $x_{s}^{*}$ and $y_{s}^{*}$. We did not apply the off-axis corrections to the Gaussian convolution model because the observed event distribution matches well with the PSF at the core without the corrections.

The modified model PSF is a combination of Equations 2 and 3, but replacing the exponential function in Equation 2 with $F_{\text {wing }}$ (Eq. 5). We compared the model PSF with the off-axis observations $\left(R>3^{\prime}\right)$ listed in Table 1 . We note that the ray-trace PSF dominates over the wing component in the inner part $\left(R \lesssim 100^{\prime \prime}\right)$ of the 2-D distribution, and we had to use the outer part $\left(\mathrm{R}>100^{\prime \prime}\right)$ where the wing component dominates in order to determine the two functions $A(\theta)$ and $B(\theta)$. Since there are not enough events above $R \sim 100^{\prime \prime}$, we did not attempt to fit the data. Instead, we find linear functions $A(\theta)$ and $B(\theta)$ by matching the 2-D contours of the observations with that of the model PSF. We find that the ray-traced PSF with the Gaussian convolution reproduces the observed 2-D distributions well at smaller radii (Fig. 4 left) and that $A(\theta)=0.025 \theta$ and $B(\theta)=1-0.025 \theta$ made the contours match at large radii (see Fig. 4 right). We note that impact of including the off-axis effect $(A(\theta)$ and $B(\theta))$ is small for the near on-axis PSF.

\subsection{Results of PSF calibration of the NuSTAR Optics}

The final model PSF includes all the modifications described above, the Gaussian convolution, the exponential wing, and the off-axis corrections for the wing component. Using the final model PSF, we fit the observed radial profiles of the near on-axis observations (see Section 3.1), and show the best-fit parameters for the Gaussian convolution width $(\sigma)$, the decay constant of the exponential wing $(a)$, and the relative normalizations $\left(C_{1} / C_{0}\right)$ of the core $\left(C_{0}\right)$ and the wing $\left(C_{1}\right)$ components for FPMA and FPMB in the top left panel of Fig. 5.

The results show that the PSF sharpens with energy; the model PSF requires smaller Gaussian width and a narrower wing, as well as a relatively larger amplitude for the core component. This trend is seen for both 

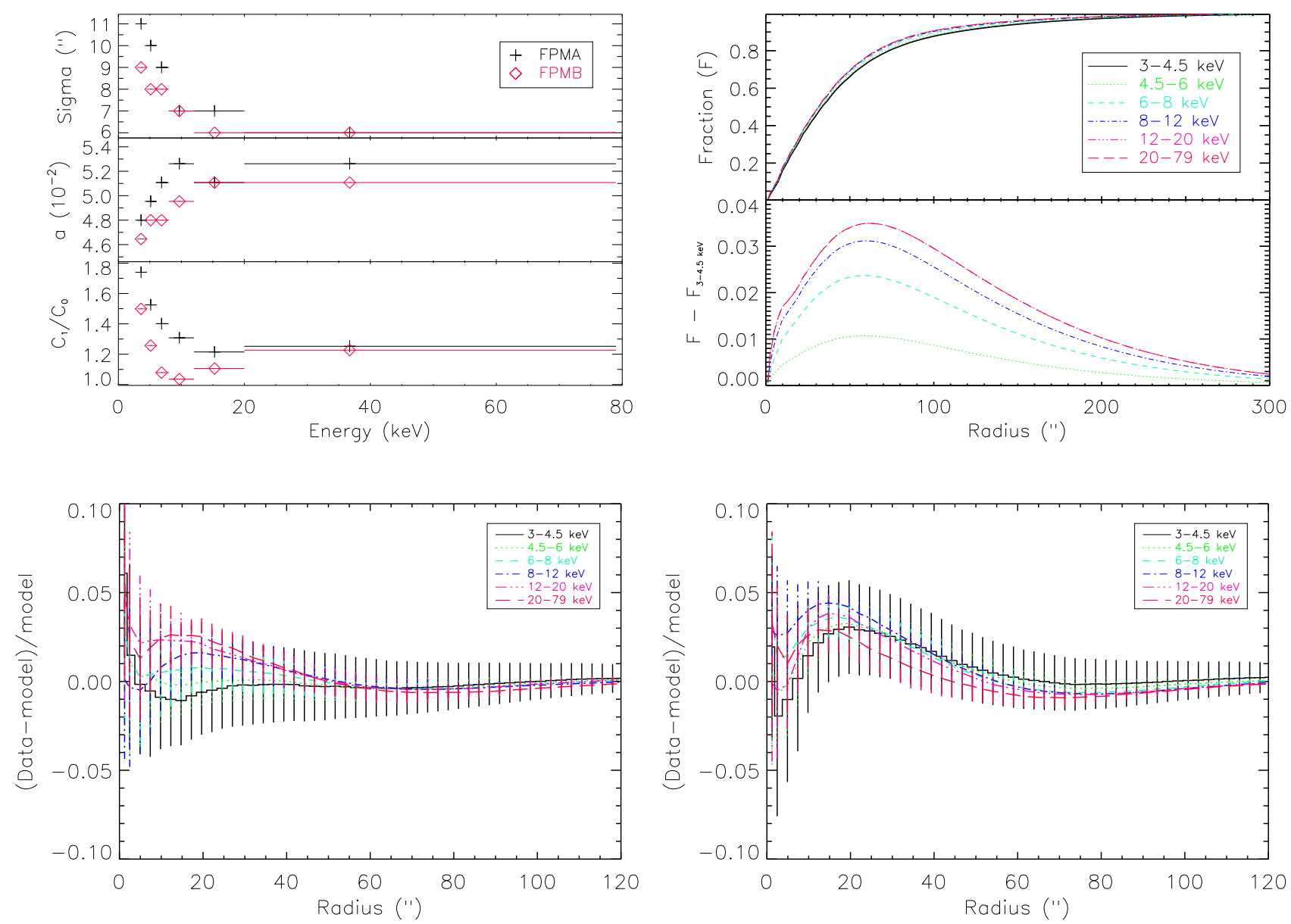

Figure 5. Top left: Measured best-fit parameters for the new PSF model with energy (see Eqs. 3 and 5). Top right: EEFs in different energy bands (top) and the differences from that in the 3-4.5 keV band (bottom) for FPMB. Bottom: Differences between the EEFs of the nine near on-axis observations and that of the PSF model for FPMA (left) and FPMB (right). The solid lines are the average difference and the error bars are the standard deviations of the nine data sets.

FPMA and FPMB and saturates at $\sim 10 \mathrm{keV}$. The reason for this could be that the inner shells have slightly better surface profiles than the outer shells do. ${ }^{7}$ It is also possible that small contamination, for example, by epoxy outgassing ${ }^{14}$ might contribute to the energy dependence. The broadening and the wing correction to the ray-traced PSF are required in all the energy bands, implying that there may be some energy independent effects as well (e.g., imperfect aspect correction and/or mirror characterization). It is not possible to further speculate the origins of the broadening and the wing with the empirical model presented in this work. More complete studies using the ray-traced model and the observatory simulator (NuSIM) ${ }^{15}$ are required.

Since the PSF shape changes with energy, the EEF for an aperture will be different for different energy bands. We measured the EEFs of the PSF model in different energy bands and show them in top right of Figure 5 . The difference is aperture dependent and is maximum between the lowest and the highest energy bands for $\sim 60^{\prime \prime}$ extraction.

Note that the best-fit parameters for the PSF model differ from observation to observation, while the parameters in top left of Figure 5 minimize the sum of the $\chi^{2}$ of the nine near on-axis observations in Table 1 . Hence, using averaged PSF for different observations will produce errors for individual observation. In order to estimate the errors, we compared the EEF of the PSF model with those of the observations, calculated the average and 
Table 2. Measured half power diameter for the on-axis observations in Table 1

\begin{tabular}{ccccc|cccc}
\hline Energy & \multicolumn{4}{|c|}{ FPMA } & \multicolumn{4}{c}{ FPMB } \\
\hline$(\mathrm{keV})$ & $\begin{array}{c}\text { HPD } \\
\left({ }^{\prime \prime}\right)\end{array}$ & $\begin{array}{c}\sigma_{\text {HPD }} \\
\left({ }^{\prime \prime}\right)\end{array}$ & $\begin{array}{c}\text { min. } \\
\left({ }^{\prime \prime}\right)\end{array}$ & $\begin{array}{c}\text { max. } \\
\left({ }^{\prime \prime}\right)\end{array}$ & $\begin{array}{c}\text { HPD } \\
\left({ }^{\prime \prime}\right)\end{array}$ & $\begin{array}{c}\sigma_{\text {HPD }} \\
\left({ }^{\prime \prime}\right)\end{array}$ & $\begin{array}{c}\text { min. } \\
\left({ }^{\prime \prime}\right)\end{array}$ & $\begin{array}{c}\text { max. } \\
\left({ }^{\prime \prime}\right)\end{array}$ \\
\hline \hline $3-4.5$ & 70.3 & 2.4 & 66.7 & 75.5 & 65.6 & 2.4 & 62.9 & 69.6 \\
$4.5-6$ & 67.1 & 1.0 & 64.7 & 67.7 & 62.6 & 1.2 & 60.9 & 64.7 \\
$6-8$ & 64.7 & 1.0 & 62.8 & 65.7 & 60.7 & 1.4 & 58.8 & 63.7 \\
$8-12$ & 63.5 & 1.1 & 61.8 & 64.7 & 59.5 & 1.5 & 57.9 & 62.8 \\
$12-20$ & 63.4 & 1.1 & 61.8 & 64.7 & 60.3 & 1.2 & 58.8 & 62.8 \\
$20-79$ & 63.4 & 1.0 & 61.8 & 64.7 & 62.4 & 1.5 & 60.8 & 65.7 \\
\hline
\end{tabular}

the standard deviation of difference between the model PSF and the data as a function of radius, and show the results in Figure 5 bottom. The difference is less than $5 \%$ for $R \gtrsim 10^{\prime \prime}$ in any energy band, and $\lesssim 3 \%$ for $R \gtrsim 60^{\prime \prime}$, implying that the inaccuracy in the effective area produced by the PSF model is $\lesssim 3 \%$ for extraction regions with $R \gtrsim 60^{\prime \prime}$. Note that the energy dependence in the top-right plot of Figure 5 is included in the PSF model and no clear trend with energy is visible in the difference plots, and thus the PSF model does not add any significant slope error to ARF for apertures of $R \gtrsim 60^{\prime \prime}$. However, we find systematic residuals for FPMB at $R=10-20^{\prime \prime}$ (Fig. 5 bottom right), which can be reduced by adding another component to the PSF model of FPMB.

In table 2, we show the average, standard deviation, minimum and maximum of HPD measured with the nine near on-axis observations in different energy bands. Note that those are observed HPD and may have been blurred by some effects such as imperfect aspect reconstruction, and incomplete background modeling, and thus not the intrinsic instrumental HPD. We find that HPD of the NuSTAR optics varies from $60^{\prime \prime}$ to $70^{\prime \prime}$ depending on the optics module and the energy band, and that the PSF of FPMB has smaller HPD, which can also be inferred from the PSF model parameters in top left of Figure 5; PSF at higher energy and of FPMB requires a sharper core.

\subsection{PSF Stability over Time}

NuSTAR hard X-ray optics are composed of glass, graphite spacer and epoxy. ${ }^{4}$ Although the materials are carefully chosen so that the coefficients of thermal expansion match well with one another, the composite structure is subject to change with temperature; for example, epoxy is known to creep with time by a thermal effect called
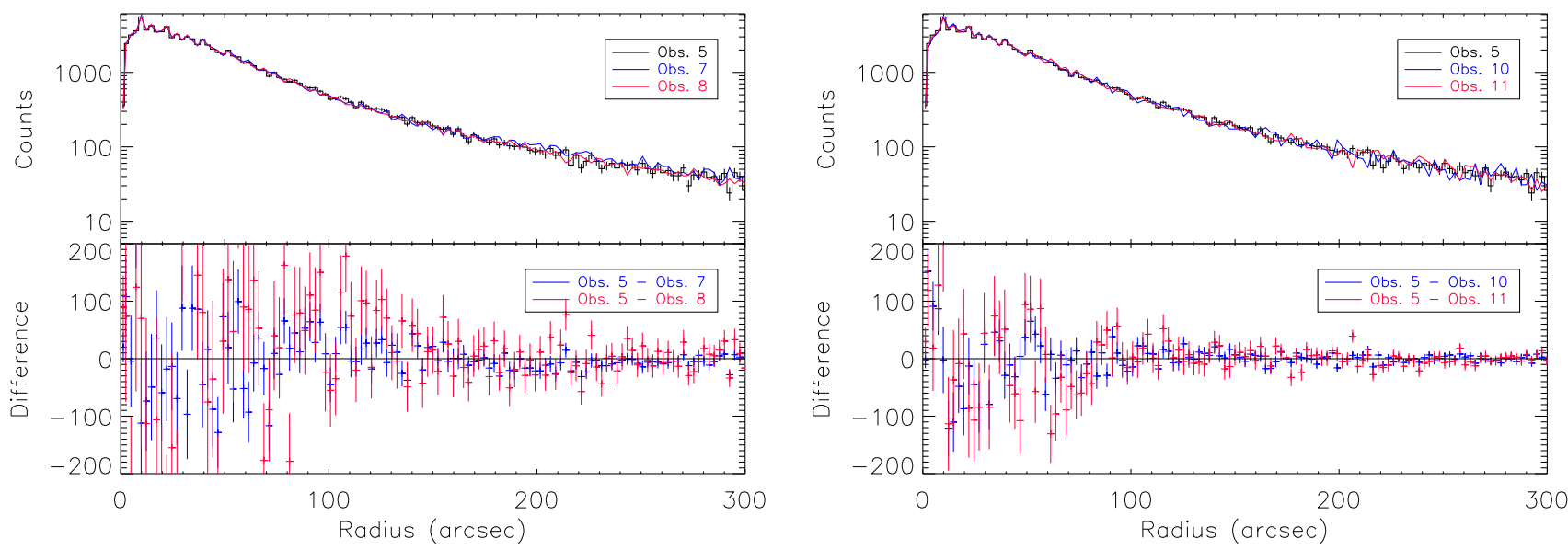

Figure 6. Left: Observed FPMA radial profiles (top) for observations 5, 7 and 8 in the $3-4.5$ keV band over $\sim 80$ days and the differences with $1 \sigma$ statistical-errors (bottom). Right: Same for observations 5, 10, and 11 over $\sim 300$ days. The profile at $T=0$ is shown in black, and those measured at later times are shown in blue and red. 
viscoelastic creep. In particular, temperature gradients applied to the optics by the Sun may have significant impact on the optics structure. As the structure of the optics may change, the PSF may broaden over time. In addition, outgassed epoxy molecules can stick to the mirror surface and scatter the incident X-rays.

Thermal effects and the epoxy outgassing on NuSTAR hard X-ray optics have been intensively studied with ground experiments using prototype flat or Wolter-I optics. The prototype optics underwent a thermal cycle or were exposed to large epoxy outgassing, and the surface profiles and X-ray scattering properties of the optics were measured before and after the experiments, where it was shown that the structure of $N u S T A R$ optics will be stable at least over $\sim 10$ years ${ }^{14}$ hence no significant change in PSF is expected over the period. We verify this by comparing radial profiles of point source observations (Table 1 ) taken $\sim 80$ days and $\sim 300$ days apart.

We produced radial profiles of the observations 5, 7, 8, 10, and 11 (Table 1) in the energy bands used above. In order to see any change in PSF, we subtracted the late-time profiles ( $T \sim 80$ and 300 days, observations 7,8 , 10 and 11) from the reference profile $(T=0$, observation 5$)$. The radial profiles and the differences in the $3-4.5$ $\mathrm{keV}$ are shown in Figure 6, which show no significant difference between the reference and the late-time profiles, considering difference between profiles of two temporally adjacent observations. The results are similar in the other energy bands as well. We therefore conclude that there was no significant change in the PSF.

\section{CONCLUSION}

We calibrated the PSF of the NuSTAR hard X-ray optics using point source observations. We show that the current ray-traced PSF alone is not able to describe the observed event distributions. In order to explain the observed distributions, we convolved the ray-trace PSF with a Gaussian function, added an extra wing component with small off-axis correction factors using empirical and analytic functions. The modified PSF model describes the observations well, and the error in the effective area produced by the PSF model is measured to be $\lesssim 3 \%$ for extraction apertures with $R \gtrsim 60^{\prime \prime}$. The PSF model described in this paper is included in the NuSTAR CALDB versions 20131007 and later. We find that the PSF of the NuSTAR optics changes with energy, being sharper at higher energies. Full ray-trace PSF modeling and NuSTAR observatory simulator need to be used for understanding the energy dependent behavior. Finally, we show that angular response of NuSTAR optics has been stable over the period of $\sim 300$ days between 2012 July and 2013 April. Further studies will be conducted to improve the accuracy of the PSF model and monitor the stability of the PSF.

\section{ACKNOWLEDGMENTS}

This work was supported under NASA Contract No. NNG08FD60C, and made use of data from the NuSTAR mission, a project led by the California Institute of Technology, managed by the Jet Propulsion Laboratory, and funded by the National Aeronautics and Space Administration. We thank the NuSTAR Operations, Software and Calibration teams for support with the execution and analysis of these observations. This research has made use of the NuSTAR Data Analysis Software (NuSTARDAS) jointly developed by the ASI Science Data Center (ASDC, Italy) and the California Institute of Technology (USA).

\section{REFERENCES}

[1] Harrison, F. A., Craig, W. W., Christensen, F. E., and et al., "The Nuclear Spectroscopic Telescope Array (NuSTAR) High-energy X-Ray Mission," ApJ. 770, 103 (2013).

[2] Madsen, K. K., Harrison, F. A., Boggs, S. E., and et al., "The Nuclear Spectroscopic Telescope Array (NuSTAR) High-energy X-ray Mission," Proc. SPIE 9144 (2014).

[3] Serlemitsos, P. J., Jalota, L., Soong, Y., and et al., "The X-ray telescope on board ASCA," PASJ. 47, 105-114 (1995).

[4] Hailey, C. J., An, H., Blaedel, K. L., and et al., "The Nuclear Spectroscopic Telescope Array (NuSTAR): optics overview and current status," Proc. SPIE 7732, 77320T (2010).

[5] Zhang, W. W., "Manufacture of mirror glass substrates for the NuSTAR mission," Proc. SPIE 7437, 74370N (2009). 
[6] Christensen, F. E., Jakobsen, A. C., Brejnholt, N. F., and et al., "Coatings for the NuSTAR mission," Proc. SPIE 8147, 81470U (2011).

[7] Craig, W. W., An, H., Blaedel, K. L., and et al., "Fabrication of the NuSTAR flight optics," Proc. SPIE 8147, 81470H (2011).

[8] Brejnholt, N. F., Christensen, F. E., Jakobsen, A. C., and et al., "NuSTAR ground calibration: The Rainwater Memorial Calibration Facility (RaMCaF)," Proc. SPIE 8147, 81470I (2011).

[9] Koglin, J. E., An, H., Barrière, N., and et al., "First results from the ground calibration of the NuSTAR flight optics," Proc. SPIE 8147, 81470J (2011).

[10] Westergaard, N. J., "MT_RAYOR: A versatile raytracing tool for x-ray telescopes," Proc. SPIE 8147, 1311 (2011).

[11] Wik, D. R., Hornstrup, A., Molendi, S., and et al., "NuSTAR Observations of the Bullet Cluster: Constraints on Inverse Compton Emission," ArXiv e-prints, ArXiv:1403.2722 (2014).

[12] van Speybroeck, L. P. and Chase, R. C., "Design parameters of paraboloid-hyperboloid telescopes for X-ray astronomy.," Appl. Opt. 11, 440-445 (1972).

[13] Spiga, D., Cotroneo, V., Basso, S., and Conconi, P., "Analytical computation of the off-axis effective area of grazing incidence X-ray mirrors," A $A \& A$ 505, 373-384 (2009).

[14] An, H., Christensen, F. E., Doll, M., and et al., "Evaluation of epoxy for use on NuSTAR optics," Proc. SPIE 7437, 74371J (2009).

[15] Harp, D. I., Liebe, C. C., Craig, W., Harrison, F., Kruse-Madsen, K., and Zoglauer, A., "NuSTAR: system engineering and modeling challenges in pointing reconstruction for a deployable x-ray telescope," Proc. SPIE 7738, 77380Z (2010). 